\title{
Non-conformally flat bulk spacetime and the 3-brane world
}

\author{
Parampreet Singh ${ }^{a}$ and Naresh Dadhich ${ }^{b}$ \\ Inter-University Centre for Astronomy and Astrophysics, Post Bag 4, Ganeshkhind, Pune 411 007, India.
}

\begin{abstract}
We show that for non-conformally flat bulk spacetime, there exist no bound modes for zero mass graviton on the 3-brane. The brane world model is therefore unstable for the bulk spacetime being different from the conformally flat anti - de Sitter space.
\end{abstract}

PACS: 04.50.+h, 11.10.Kk

Inspired by the requirement of dimensions more than four for the string theory, the study of gravity in higher dimensions has been in vogue for quite a while. Generally the extra dimensions are taken to be compact and could be probed only at the Planck energy. In view of the recent work, it is no longer necessary to have the extra dimensions compact, they could be large with matter fields being confined to a 3-brane in $1+3+d$ dimensional bulk $[1,2]$ and gravity propagating in the extra dimensions. The brane world model envisages that the standard model matter fields live on the 3-brane while gravity propagates in the bulk which is the $Z_{2}$ symmetric Einstein space. In particular, the most talked of Randall-Sundrum (RS) model [3] has 5-dimensional anti - de Sitter space as the bulk with brane being Minkowskian flat. It is then shown that gravity can, even with infinite fifth dimension, be localized on a single 3-brane.

In the RS model, the confinement of zero mass graviton has been obtained by the exponential conformal factor in the non-factorizable bulk metric given by

$$
d s^{2}=d y^{2}+e^{-2 k|y|}\left(-d t^{2}+d \mathbf{x}^{2}\right)
$$

This is the anti - de Sitter space in the 5-dimensional bulk for $y \neq 0$ with $\Lambda=-6 k^{2}$. The brane is sitting at the fixed point $y=0$ with $y<0$ being identified by $y>0$ reflecting the $Z_{2}$ symmetry. The perturbations of the metric do give on the brane the Newtonian $1 / r$ potential with the correction term going as $1 / r^{3}$. Thus the conventional gravity is recovered on the brane with the first order correction which could be probed only at sub-millimeter level [4].

Recently there have been many works questioning the stability of the brane world model in various contexts [5]. In particular it has been argued that there has to be maintained a very delicate balance between the $\Lambda$ and the tension in the brane [6]. In the above metric, the brane is flat while the bulk is anti - de Sitter. These are undoubtedly very special spaces describing very specific idealized situations and hence could by no means be considered generic. Recently, it has also been shown that the bound state of zero mass graviton is normalizable only when the brane spacetime could at best be Ricci flat for spherically symmetric spacetimes. That is, the brane space cannot be different from the Schwarzschild solution, not even a de Sitter space [7]. Thus localizability of gravity on the brane is accomplished under very special circumstances.

In this note, we wish to examine what happens if we take the bulk to be a non conformally flat Einstein space with of course negative $\Lambda$. That is it has non-zero Weyl curvature which would induce a trace free stress tensor on the brane. The question is, would there exist a bound state for the zero mass graviton in this case? It turns out that it doesn't. That is what we show in the following.

It may be noted that one of the most attractive features of the brane world model was to have free gravitational field in the bulk reflected on the brane through the projection of the Weyl curvature as trace free matter field on the brane. This is how the bulk back reacts to produce "tidal" charge on the brane which gets however washed out when the bulk is anti - de Sitter because it has vanishing Weyl curvature (conformally flat). It is therefore pertinent to consider the solution of the the equation $G_{a b}=-\Lambda g_{a b}$ for the bulk spacetime with non-zero Weyl curvature. There exists such a solution obtained due to Nariai [8] for the 4-dimensional Einstein space, which is conformally non flat. We extend this solution to the 5-dimensional bulk spacetime and it reads as follows:

$$
d s^{2}=d y^{2}+e^{-2 k|y|}\left(-d t^{2}+d r^{2}\right)+\frac{1}{2 k^{2}}\left(d \theta^{2}+\sinh ^{2} \theta d \phi^{2}\right) .
$$

This is the solution of the equation,

$$
G_{a b}=-\Lambda g_{a b}
$$

with $\Lambda=-3 k^{2}$. The projection of the Weyl curvature on the brane is given by [9]

$$
E_{\mu \nu}:=C_{\mu \rho \nu \sigma} n^{\rho} n^{\sigma}
$$


where $n^{\alpha}$ is the unit normal, and we have

$$
E_{\mu \nu}=\left(E_{t t}, E_{r r}, E_{\theta \theta}, E_{\phi \phi}\right)=-\frac{k^{2}}{2}\left(g_{t t}, g_{r r},-g_{\theta \theta},-g_{\phi \phi}\right) .
$$

Here the latin indices run from $0, \ldots, 4$ while the greek from $0, \ldots, 3$. Note that the brane $y=0$ is not flat in this case and could describe a cloud of string dust of uniform energy density [10]. Since the brane metric is not a vacuum, one might expect from the investigation of ref.[7] that gravity may not be localized in this case. But the metric (2) is not in the standard RS form as only the non-angular part of the metric shares the conformal factor. This result would therefore not follow straightway. Our metric is spatially anisotropic which is essential for the Weyl curvature to be non-zero. If the conformal factor is shared isotropically, it would trivially be conformally flat.

We would now study the perturbations of the above metric for finding the bound state of massless graviton. In order to find a massless ground state mode with two degrees of freedom we will do the linear perturbative analysis of the above metric. We write the perturbed metric, $\tilde{g}_{a b}=g_{a b}+h_{a b}$. Since our main objective is to examine existence of the bound normalizable massless mode on the brane, we take the metric fluctuations in the extra dimension to vanish, i.e.

$$
h_{t y}=h_{r y}=h_{\theta y}=h_{\phi y}=h_{y y}=0
$$

We further impose the following gauge conditions on the metric fluctuations on the brane

$$
\nabla^{\mu} h_{\mu \nu}=0, \quad h_{\mu}^{\mu}=0 .
$$

We then obtain the equation

$$
\square h_{a b}=A_{a b, i}^{i}+\Gamma_{i j}^{i} A_{a b}^{j}-\Gamma_{a j}^{i} A_{i b}^{j}-\Gamma_{b j}^{i} A_{i a}^{j}=\Lambda h_{a b}
$$

where $\Lambda$ is the cosmological constant in the bulk and

$$
A_{a b}^{i}:=g^{i j}\left(h_{a b, j}-\Gamma_{a j}^{k} h_{k b}-\Gamma_{b j}^{k} h_{a k}\right) .
$$

Here the cosmological constant in the bulk is related to $k$ by $\Lambda=-3 k^{2}$. We look for the solutions of the form

$$
h_{a b}=e_{a b} \Psi(y) e^{i p_{j} x^{j}}
$$

and further assume that the the polarization tensor $e_{a b}$ is orthogonal to a unit timelike vector $u^{a}=(1,0,0,0,0)$ i.e. $e_{a b} u^{b}=0$. It is interesting to note that our form of the metric clearly demarcates the physical modes of propagation. This happens because propagation vector is null which only allows $h_{\theta \theta}$ and $h_{\theta \phi}$ as the physical modes, where $h_{\theta}^{\theta}=-h_{\phi}^{\phi}$ due to traceless condition. It is easy to check that other modes are ruled out because the null form of $p_{a}$ demands that $y$ should be constant.

The gauge conditions impose constraints on the derivatives of $h_{\theta \theta}$ and $h_{\theta \phi}$ which are

$$
h_{\theta \phi, \phi}+\sinh ^{2} \theta h_{\theta \theta, \theta}+\sinh 2 \theta h_{\theta \theta}=0
$$

and

$$
h_{\theta \phi, \theta}-h_{\theta \theta, \phi}+\operatorname{coth} \theta h_{\theta \phi}=0 .
$$

Solving the wave equation and using $m^{2}$ as a constant for separation of variables it can be shown that the $y$ part comes out to be

$$
\Psi^{\prime \prime}(y)-2 k \Theta(y) \Psi^{\prime}(y)+\left(4 k^{2}+m^{2} e^{2 k|y|}\right) \Psi(y)=0
$$

where $\Theta(x)$ is the unit step function,

$$
\Theta(x)= \begin{cases}+1 & \text { if } x>0 \\ -1 & \text { if } x<0\end{cases}
$$

Eq.(13) is not in the form of a wave equation, however we can always transform it into that form through the following substitution 


$$
\Psi(y)=\chi(y) \psi(y), \quad \chi(y)=\exp \left(k \int_{-\infty}^{\infty} \Theta(x) d x\right) .
$$

On this substitution the first derivative term in eq.(13) vanishes and we obtain

$$
\psi^{\prime \prime}(y)+\left[2 k \delta(y)+3 k^{2}+m^{2} e^{2 k|y|}\right] \psi(y)=0 .
$$

Putting $m=0$ we obtain the required wave equation which would tell us about the effect of extra dimension on the massless modes of the metric fluctuations on the brane,

$$
\psi^{\prime \prime}(y)+\left(2 k \delta(y)+3 k^{2}\right) \psi(y)=0 .
$$

The presence of Dirac delta function in the above equation tells us that we should look for the solutions which are the functions of $|y|$. The above equation has an irregular singular point at $y=0$, hence in order to look for its solution let us consider a general equation of the form

$$
f^{\prime \prime}(y)+(a \delta(y)+b) f(y)=0
$$

and look for solutions $f=f(|y|)$. Then it can be easily seen that the solution would exist only if $a^{2}=-4 b$ with $f=\exp (-a|y| / 2)$.

The wave equation describing $y$ dependence of massless mode of the metric fluctuation in the RS model is given by

$$
\psi^{\prime \prime}(y)+\left(4 k \delta(y)-4 k^{2}\right) \psi(y)=0 .
$$

With $a=4 k$ and $b=-4 k^{2}$ the RS equation satisfies the constraint $a^{2}=-4 b$ and gives the ground state wavefunction as $\exp (-2 k|y|)$. However, in our case, eq.(16) yields $a=2 k$ and $b=3 k^{2}$ which implies that the constraint condition is not satisfied and hence there can exist no solution of the form $f=f(|y|)$.

We would like to argue that a brane world model should admit all solutions of the Einstein space equation in the bulk and not only the specific anti - de Sitter solution. The really interesting situation would be presented by the conformally non flat bulk solution which would project on the brane free gravitational field as trace free matter giving rise to "tidal" charge which would make non trivial contribution. It is a pity that the most exciting aspect of the model is not realizable in the present form of its formulation. It may be recalled that an interesting solution in terms of the Reissner-Nordstrom (RN) metric was obtained for a black hole on the brane [11] precisely due to the contribution of the bulk Weyl curvature on the brane. It turns out that the Nariai metric (2) projects on the brane the stresses precisely of the form for which the RN metric is the exact solution. Thus the Nariai metric (2) gives the bulk spacetime metric corresponding to the black hole solution [11] on the brane.

We have thus shown that the brane model described by eq.(2) does not allow the existence of the bounded massless mode. Since that does not happen there is no question of recovering the $1 / r$ Newtonian potential between two massive particles on the brane. That means a non conformally flat bulk spacetime with negative $\Lambda$ does not yield the conventional gravity in the 4-dimensions on the brane. At the same time our metric is an exact good solution of the Einstein space equation in the bulk. This is however hardly surprising once we notice the kind of fine tuning which is required between $a$ and $b$ to solve an ill behaved differential equation like eq.(17). The RS metric is just an example of that in which the parameters just fit perfectly. Any deviation from the specific matching values would lead to non-confinement as is clear from eq.(17). The brane world model thus seems to be very brittle for inclusion of non-zero Weyl curvature in the bulk.

By studying gravity on the branes through the 4-dimensional discontinuity caused by the $Z_{2}$ symmetric bulk, it has been shown that if the bulk is an Einstein space near the brane, Einstein's equation could approximately be recovered on the brane. On the other hand if it is required to be exactly the anti - de Sitter space, Einstein's equation cannot hold even approximately unless the matter obeys a very contrived and artificial equation of state [12]. And we show that if it is not anti - de Sitter, gravity cannot remain confined to the brane.

Acknowledgment: We thank S. Shankaranarayanan and T. Padmanabhan for helpful discussions as well as for reading the manuscript. PS thanks Council for Scientific \& Industrial Research for grant number: 2 - 34/98(ii)E.U-II. 
ar param@iucaa.ernet.in

b nkd@iucaa.ernet.in

[1] N. Arkani-Hamed, S. Dimopolous and G. Dvali, Phys. Lett. B429, 263 (1998); I. Antoniadis, N. Arkani-Hamed and S. Dimopolous, Phys. Lett. B436, 257 (1998); N. Arkani-Hamed and S. Dimopolous, Phys. Rev. D59, 086004 (1999).

[2] G. Shiu and S. H. Henry Tye, Phys. Rev. D58, 106007 (1998).

[3] L. Randall and R. Sundrum, Phys. Rev. Lett. 83, 4690 (1999).

[4] C. D. Hoyle, et.al., Sub-millimeter tests of the gravitational inverse square law: A search for "large" extra dimensions, hepph/0011014; D. j. H. Chung, et.al, Experimental Probes of the Randall-Sundrum Infinite Extra Dimension, hep-ph/0010103.

[5] D. Marolf, M. Trodden, Black Holes and Instabilities of Negative Tension Branes, hep-th/0102135. M.G. Santos, F. Vernizzi, P.G. Ferreira, Isotropization and instability of the brane, hep-ph/0103112.P. Binetruy, J.M. Cline, C. Grojean, Phys.Lett. B489 403, (2000).

[6] T. Boehm, R. Durrer and C. van de Bruck, Dynamical Instabilities of the Randall-Sundrum Model, hep-th/0102144.

[7] T. Padmanabhan and S. Shankaranarayanan, Vanishing of cosmological constant in nonfactorizable geometry, hepth/0011159.

[8] H. Nariai, Sci. Rep. Tohoku Univ. 34, 160 (1950).

[9] T. Shiromizu, K. Maeda, M. Sasaki, Phys.Rev. D62 024012 (2000).

[10] N. Dadhich, On product spacetime with 2-sphere of constant curvature, gr-qc/0003026.

[11] N. Dadhich, R. Maartens, P. Papadopoulos and V. Rezania, Phys. Lett. B487, 1 (2000).

[12] N. Deruelle and J. Katz, Gravity on branes, gr-qc/01040007. 